\title{
Modeling and Analysis of Three Phase Stand Alone Induction Generator under Single Phase Load
}

\author{
${ }^{1}$ A. M. Alsalloum, ${ }^{2}$ A. I. Alolah, ${ }^{3}$ R. M. Hamouda \\ ${ }^{1,2,3,}$ EE Dept, College of Engineering, King Saud University, P.O. Box 800, Riyadh 11421, Saudi Arabia
}

\begin{abstract}
A model of a delta-connected three-phase stand alone induction generator (SEIG) feeding a deltaconnected load is derived and presented. This model includes the core losses where the core resistance is modeled as a function of the magnetizing reactance. Furthermore, the magnetizing reactance has been included in the negative sequence equivalent circuit as a variable. The model is applicable to $R-L, R-C$ or resistive and three phase or single phase loads, balanced or unbalanced with any degree of unbalance. The SEIG performance has been computed for different single phase loading conditions iteratively. These results are confirmed experimentally.
\end{abstract}

Keywords: induction generator, unbalance, stand alone, SEIG.

Symbols

\section{Nomenclature}

a $\quad$ Operator $1 \angle 120^{\circ}$

$R, X, Z \quad$ Resistance, reactance, and impedance respectively.

$C, X_{C} \quad$ Excitation capacitance and its reactance.

$F, v \quad$ Per unit frequency and speed respectively.

$V, I \quad$ Voltage and current respectively.

Subscripts

$0,1,2$ Zero, Positive, and negative sequence components respectively.

$s, r, m \quad$ Stator, rotor and Magnetizing quantity respectively.

$a b, b c, c a \quad$ Phases of the system.

$P, n \quad$ Positive and negative, respectively.

$g \quad$ Air gap quantity.

$c \quad$ Core quantity

$G, L \quad$ Generator and load respectively.

\section{Introduction}

Due to the increased emphasis on the energy issues and problems, concentration has been focused upon developing autonomous electric power supplies to be operated in remote and rural areas where electric services is unavailable from existing or nearby grids. These types of power sources can be used even in regions supplied by network grids in the event of power interruptions. Among such types that have received a notable attention and importance is the three-phase self-excited induction generator due to its numerous advantages such as simple design, robustness, and low installation and maintenance costs [1-4]. Experimental works and computer simulations have been extensively performed in order to model and analyze both steady state and transient performance of the SEIG under balanced operating conditions. However, the unbalanced operation of the SEIG has been given little attention despite its practical needs. There are two main methods to predict the steady state performance of the SEIG under balanced operating conditions. The first method is based on the generalized machine theory [5]. The second method is based on the analysis of the generalized per-phase equivalent circuit of the induction machine by applying either the loop impedance or the nodal admittance concept $[6,7]$. Furthermore, other studies have concentrated only on the single-phase self-excited induction generator and its voltage regulation improvement [8]. The influence of the terminal capacitance has been investigated in $[9,10]$. The previous studies have centralized mainly on modeling and analyzing the performance of SEIG under only balanced operating conditions. In this paper, modeling and performance analysis of SEIG under single phase loading conditions in the steady state are presented. A model of a delta-connected SEIG feeding a deltaconnected load is derived in detail. The effect of the machine core losses is considered by representing the core resistance as a second order polynomial in terms of $X_{m}$. Furthermore, the magnetizing reactance has been included in the negative sequence equivalent circuit as a variable. The positive and negative sequence equivalent circuits are used to model the SEIG. The final characteristic equation is reached by equating both the positivesequence and negative-sequence voltages across the SEIG and the load. 


\section{Modeling}

Fig. 1 shows a $\Delta$-connected SEIG feeding a $\Delta$-connected load. This load may be defined as follows:

$Z_{a b}=Z_{L a b} / / X_{C a b}, \quad Z_{b c}=Z_{L b d} / / X_{C b c}, \quad Z_{c a}=Z_{L c d} / / X_{C c a}$,

where,

$Z_{L a b}=R_{L a b}+j X_{L a b}, Z_{L b c}=R_{L b c}+j X_{L b c}, \quad Z_{L c a}=R_{L c a}+j X_{L c a}$

The symmetrical components for this type of load connection are as follows [10]:

$\left[\begin{array}{l}Z_{0} \\ Z_{1} \\ Z_{2}\end{array}\right]=\frac{1}{3}\left[\begin{array}{ccc}1 & 1 & 1 \\ 1 & a & a^{2} \\ 1 & a^{2} & a\end{array}\right]\left[\begin{array}{l}Z_{a b} \\ Z_{b c} \\ Z_{c a}\end{array}\right]$

Since the load as well as the generator is connected in delta, hence, the phase and the line voltages of both the generator $\left(V_{a b G}\right)$ and the load $\left(V_{a b L}\right)$ are equal. The symmetrical components of the phase voltage $\left(V_{a b}\right)$ at the load side are as follows:

$\left[\begin{array}{c}V_{a b L 0} \\ V_{a b L 1} \\ V_{a b L 2}\end{array}\right]=\left[\begin{array}{lll}Z_{0} & Z_{2} & Z_{1} \\ Z_{1} & Z_{0} & Z_{2} \\ Z_{2} & Z_{1} & Z_{0}\end{array}\right]\left[\begin{array}{c}I_{a b L 0} \\ I_{a b L 1} \\ I_{a b L 2}\end{array}\right]$

It is known that for a $\Delta$-connected load, $V_{a b L 0}=0$, so substituting in (2), yields:

$I_{a b L O}=-\left(Z_{2} I_{a b L 1}+Z_{1} I_{a b L 2}\right) / Z_{0}$

Also from (2):

$V_{a b L 1}=Z_{1} I_{a b L O}+Z_{0} I_{a b L 1}+Z_{2} I_{a b L 2}$

$V_{a b L 2}=Z_{2} I_{a b L O}+Z_{1} I_{a b L 1}+Z_{0} I_{a b L 2}$

From Fig.2, the terminal voltages of the SEIG are:

$$
\begin{aligned}
& V_{p}=-I_{a b 1} Z_{p} \\
& V_{n}=I_{a b 2} Z_{n}
\end{aligned}
$$

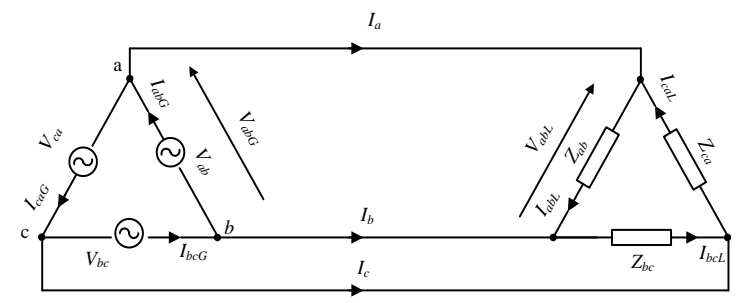

Fig. 1. $\Delta$-connected SEIG feeding a $\Delta$-connected load

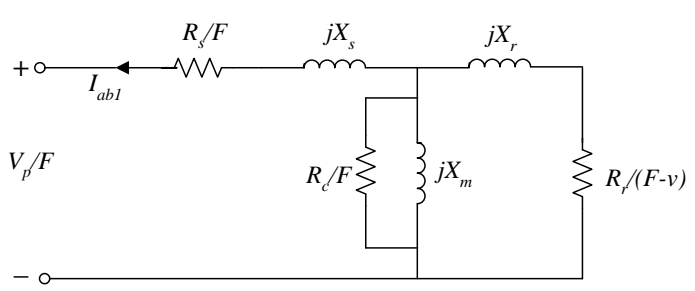

(a)

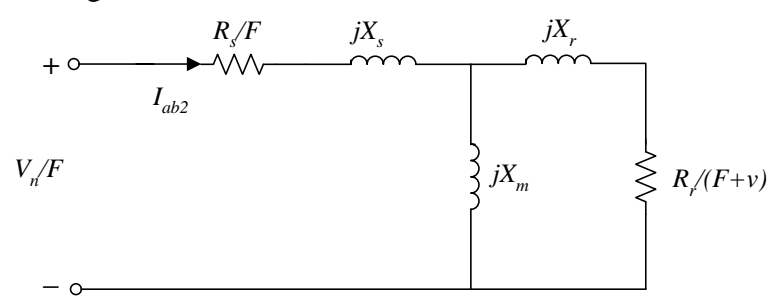

(b)

Fig. 2. Sequence equivalent circuits of SEIG

(a) Positive-sequence (b) Negative-sequence 
It can be shown that the sequence components of phase and line currents shown in Fig.1 are related as follows [10]:

$$
\begin{aligned}
& I_{a 1}=(1-a) I_{a b 1} \\
& I_{a 2}=\left(1-a^{2}\right) I_{a b 2}
\end{aligned}
$$

Since self-excitation is assumed to occur, i.e., $I_{a l} \neq 0$ and using equations 3-9, yields: $A_{1} A_{2}-A_{3} A_{4}=0$

where $\quad A_{1}=\left(\frac{Z_{1} Z_{2}}{Z_{0}}\right)-Z_{0}-Z_{p} \quad A_{2}=Z_{0}-\left(\frac{Z_{1} Z_{2}}{Z_{0}}\right)+Z_{n} \quad A_{3}=Z_{2}-\left(\frac{Z_{1}^{2}}{Z_{0}}\right) \quad A_{4}=\left(\frac{Z_{2}^{2}}{Z_{0}}\right)-Z_{1}$

This characteristic equation is complex. Accordingly, both real and imaginary parts must equal to zero. Substituting the machine parameters, speed, and excitation capacitors values, yields two nonlinear equations with constant coefficients in $F$ and $X_{m}$. Solving iteratively to find the real roots of the equations that satisfy the constraints, the values of $F$ and $X_{m}$ can be found. Hence, the performance of the generator may be determined. The derived model was tested against the machine with the parameters mentioned in appendix A.

\section{IV. $\quad$ Results and Discussion}

\subsection{Three Excitation Capacitors-Single Phase Load}

The SEIG is excited by a balanced set of excitation capacitors and a single phase resistive load is attached to it, namely, $R_{a b}=5.3$ p.u. In Fig.3, the variation of the magnetizing reactance $X_{m}$ against the excitation capacitance for this loading condition is shown along with the variation of the positive-sequence equivalent circuit terminal voltage $V_{p}$, the air gap voltage $V_{g}$, and the frequency $F$. It is clear that $X_{m}$ varies with the excitation capacitance in a nonlinear fashion as expected. Furthermore, the voltage $V_{p}$ has a maximum at a certain capacitance and the same applies for $V_{g}$. On the other hand, the frequency $F$ decreases as the excitation capacitance is increased. Fig. 4 shows the variation of phase (line) voltages with excitation capacitance. The output voltages are unbalanced as expected where $V_{b c}$ is the highest among all output voltages and $V_{c a}$ is the lowest. Furthermore, Fig.5 shows the variation of line currents against the excitation capacitance. The line currents are unbalanced where $I_{a}$ is the highest and $I_{b}$ is the lowest.

\subsection{Two Excitation Capacitors-Single Phase Load}

The SEIG is excited by $C_{b c}$ and $C_{c a}$ with a single phase load attached to its terminals. The load consists of $R_{L}=5.3$ p.u.. Fig. 6 shows the variation of $V_{p}, V_{g}, F$, and $X_{m}$ with the excitation capacitance. Again we can say that these parameters behave in similar manner as those of the previous case. Fig. 7 shows the variation of output phase (line) voltages against excitation capacitance. The solid line traces represent the output voltages when the load is attached across phases $(b)$ and $(c)$, i.e., parallel to one of the excitation capacitors. Furthermore, the dashed line traces represent the output voltages when the load is attached across phases $(a)$ and $(b)$. The output voltages are unbalanced as expected where the degree of unbalance depends on the load location with in the system. On the other hand, Fig. 8 shows the variation of line currents for the same situations described earlier. The line currents are unbalanced as expected and Line (a) as well as line (c) is carrying higher current when the load is connected across phases $(a)$ and $(b)$.

\subsection{Single Excitation Capacitor-Single Phase Load}

The SEIG is excited by a single capacitor, namely, $C_{b c}$ with a 5.3 p.u. attached to its terminals. Fig. 9 and Fig. 10 show the variation of output phase (line) voltages and the variation of line currents against excitation capacitance, respectively. The solid line traces represent the output voltages (currents) when the load is attached across phases $(b)$ and $(c)$, i.e., parallel to the excitation capacitor. Furthermore, the dashed line traces represent the output voltages (currents) when the load is attached across phases $(a)$ and $(b)$. The output voltages are unbalanced with higher output voltages when the load is connected across phases $(b)$ and $(c)$. Moreover, the line currents are unbalanced with lines $(b)$ and $(c)$ carrying higher currents when the load is connected across phases $(b)$ and $(c)$. Hence, the level as well as the degree of unbalance of output voltages and line currents depends on the load location within the system.

\section{Conclusions}

A model of a delta connected SEIG feeding a delta connected load based on the sequence equivalent circuits of the SEIG and the sequence components of the three-phase load was developed. The performance of the SEIG was determined for single phase load condition. The operating conditions were found by solving the proposed 
model iteratively. The position of the single phase load affects the level and the degree of unbalance of the output voltages and currents.

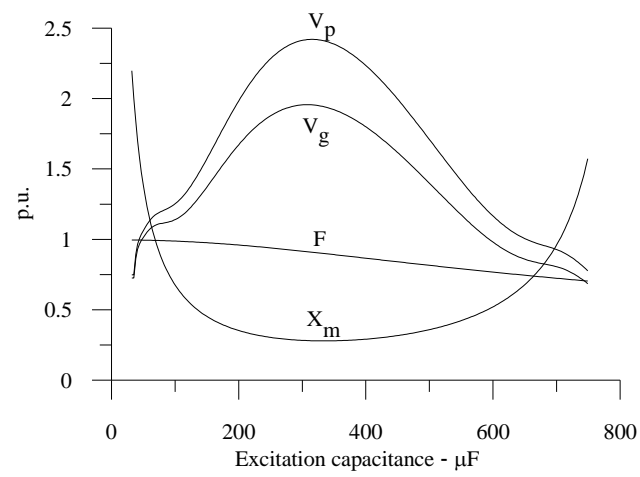

Fig. 3. Variation of $V_{p}, V_{g}, F$, and $X_{m}$ for balanced excitation capacitors and single phase load

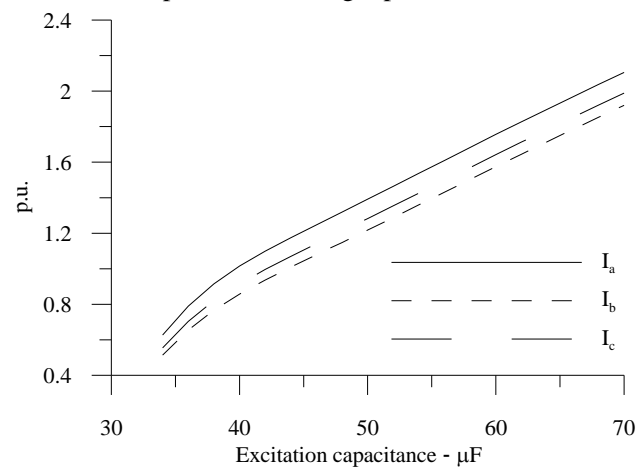

Fig. 5. Variation of line currents for balanced excitation capacitors and single phase load

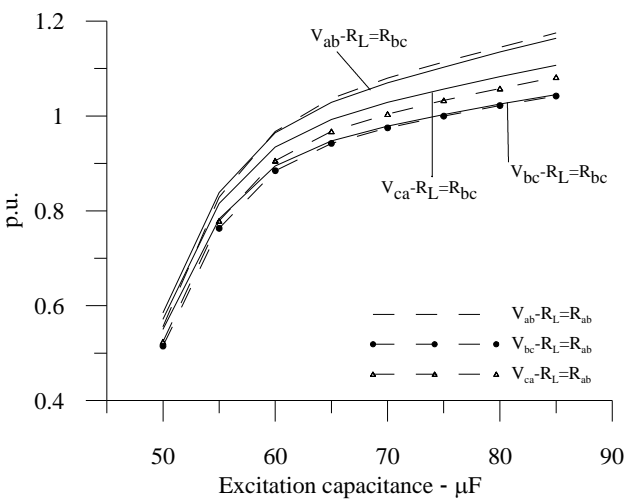

Fig. 7. Variation of phase (line) voltages for two excitation capacitors and single phase load

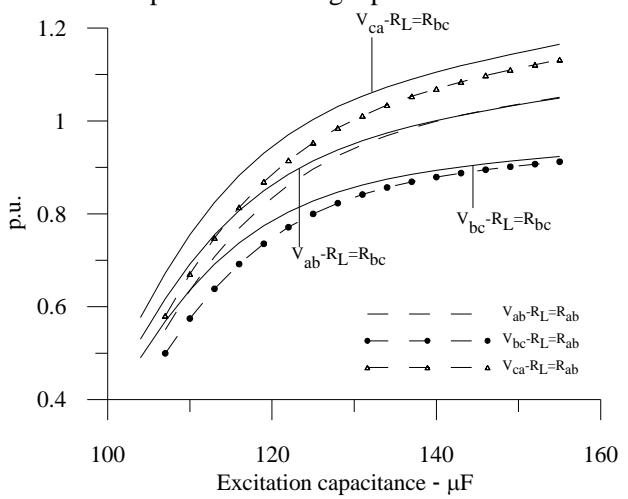

Fig. 9. Variation of phase (line) voltages for single excitation capacitor and single phase load

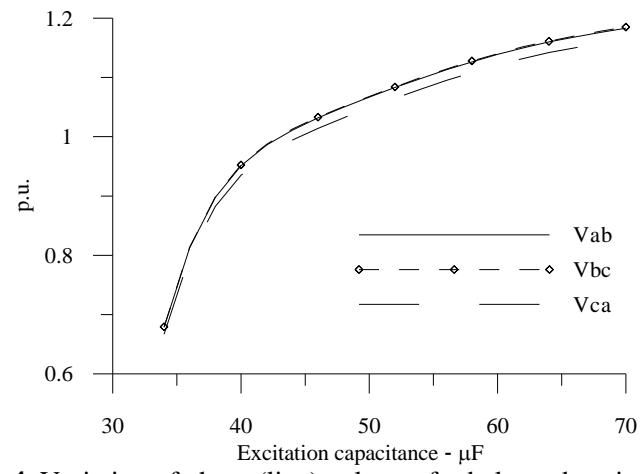

Fig. 4. Variation of phase (line) voltages for balanced excitation capacitors and single phase load

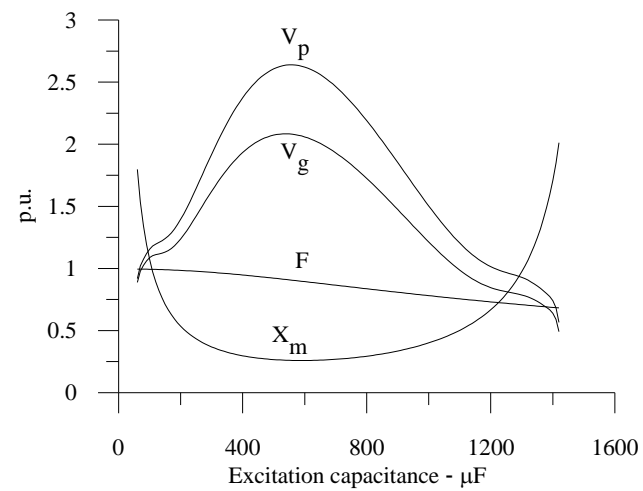

Fig. 6. Variation of $V_{p}, V_{g}, F$, and $X_{m}$ for two excitation capacitors and single phase load

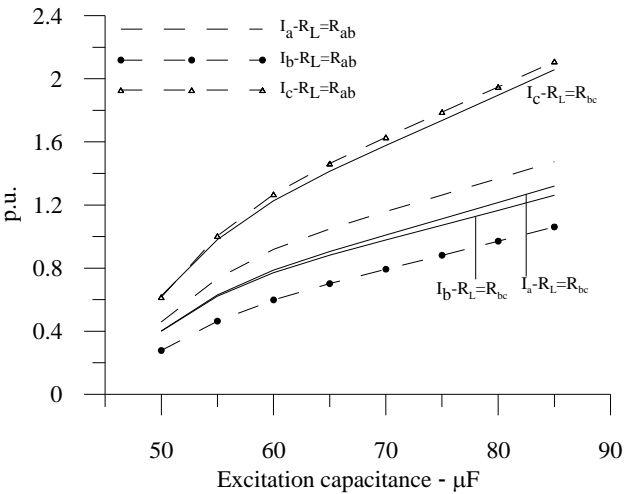

Fig. 8. Variation of line currents for two excitation capacitors and single phase load

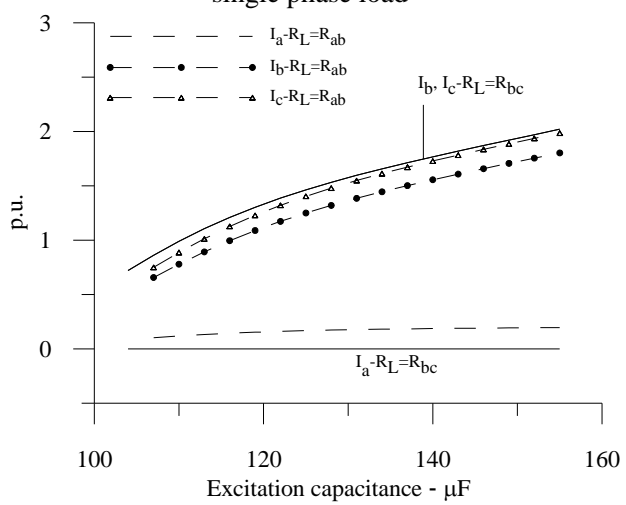

Fig. 10. Variation of line currents for single excitation capacitor and single phase load 


\section{References}

[1] Mekkaoui, N., Nait-Said, M.-S., Drid, S.," Steady-state analysis of Self -Excited Induction Generator", in Proc. International Conference on communications, Computing and Control Applications (CCCA), pp. 1-5, 2011.

[2] Elder, J., Boys, J., and Woodward, J., "The Process of Self Excitation in Induction Generators", ibid, (130), pt. B., No. 2, pp. 103$107,1983$.

[3] Haque, M. H.," A Novel Method of Evaluating Performance Characteristics of a Self-Excited Induction Generator ", IEEE Trans., EC-24(2), pp. 358-365, 2009.

[4] Raina, G., and Malik, O., "Wind Energy Conversion Using a Self-Excited Induction Generator", ibid., Power System Apparatus, Vol. PAS-102(12), pp. 3933-3936, 1983.

[5] Grantham, C., Sutanto, D., and Mismail, B., "Steady State and Transient Analysis of Self-Excited Induction Generators", IEE Proc., pt. B, (136), No. 2, pp. 61-68, 1989.

[6] Malik, N., and Mazi, A., "Capacitance Requirements for Isolated Self-Excited Induction Generators", IEEE Trans., EC-2(1), pp. 6269, 1987.

[7] Al-Jabri, A., and Alolah, A., "Capacitance Requirements for Isolated Self-Excited Induction Generators", IEE Proc., (137), pt. B, No. 3, pp. 154-159, 1990.

[8] Murthy, S.S., Kalla, U.K., Bhuvaneswari, G.," A Novel Electronic Controller Implementation for Voltage Regulation of Single Phase Self-Excited Induction Generator", in Proc. IEEE Industry Applications Society Annual Meeting (IAS), pp. 1-8, 2010.

[9] Tutkun, N., Arslsn, F.," Determination of capacitance range in the self-excited induction generator through the hybrid genetic algorithms ", in Proc. International Symposium on Power Electronics Electrical Drives Automation and Motion (SPEEDAM), pp. 1613-1617, 2010.

[10] Phumiphak, P., Chat-uthai, C.," Optimal capacitances compensation for short-shunt self-excited induction generator under inductive load", in Proc. International Conference on Electrical Machines and Systems (ICEMS), pp. 1-5, 2009.

[11] Alsalloum A. M., "Modeling and Performance Analysis of unbalanced Three-Phase Self-Excited Induction Generator", PhD Thesis, King Saud University, Riyadh, Saudi Arabia, 2011.

Parameters of the self-excited induction generator:

\section{Appendix A}

The test machine was a three-phase, Y/ $\Delta, 460 / 265 \mathrm{~V}, 7.1 / 12.3 \mathrm{~A}, 60 \mathrm{HZ}$, induction machine with the following parameters: $R_{s}=0.085, R_{r}=0.042, X_{s}=0.075, X_{r}=0.112, X_{u}=2.176$ (all in p.u.), $Z_{\text {base }}=37.406 \Omega$, base speed = $1800 \mathrm{rpm}$. The relation between air gap voltage and magnetizing reactance as well as the relation between core resistance and magnetizing reactance is given in [11]. 